\title{
The interaction of spatially modulated vortex pairs with free surfaces
}

\author{
By CHRISTIAN E. WILLERT $\dagger$ AND MORTEZA GHARIB \\ Graduate Aeronautical Laboratories, California Institute of Technology, Pasadena, \\ CA 91125, USA
}

(Received 19 September 1995 and in revised form 18 April 1997)

Spatially modulated vortex pairs were generated below a free surface by two counterrotating flaps whose edges approximate a sinusoid. The surface interactions of the vertically approaching vortex pairs were visualized by the shadowgraph technique. Two limiting cases were investigated in detail: the interaction with a surfactant-rich (contaminated) surface and with a surfactant-poor ('clean') surface. In the latter case shadowgraph images showed that the underlying vortex core formed a line of circular surface depressions. Subsequent measurements of the temporally evolving velocity fields using digital particle image velocimetry (DPIV) of the vortex pair cross-sections and the subsurface plane confirmed the connection process of the main vortex core with the surface. As a result of the connection the initially modulated vortex tube was broken into a line of U-vortices. In the presence of surfactants this connection could not be observed; rather a Reynolds ridge (or stagnation line) was formed and a very weak connection of the secondary separation vortex could be seen in the shadowgraphs as well as measured with the time-resolved DPIV technique.

A prerequisite for connection of the vortex with the surface is that the flow's kinematics force the vortex core, that is, regions of concentrated vorticity, toward the surface. The ensuing locally concentrated viscous flux of surface-parallel vorticity through the surface is balanced by a local surface deceleration. Surface-normal vorticity appears on each side of the decelerated region whose gradually increasing circulation is directly balanced by the loss of circulation of the surface-parallel vortex. However, the shear forces caused by small amounts of surface contamination and its associated subsurface boundary layer inhibit the connection process by preventing the essential viscous flux of parallel vorticity through the surface. Instead, the subsurface boundary layer is associated with a flux of parallel vorticity into the surface which then concentrates into the observable secondary separation vortex.

\section{Introduction}

This study on vortex/free surface interaction is motivated by the need to gain a physical understanding of the flow around and aft of ships. There are numerous observations of the wakes behind ships in the open ocean where the centreline wake can be seen in sun-glint images and in synthetic aperture radar imagery from aircraft

$\dagger$ Present address: Institute of Fluid Mechanics, German Aerospace Research Establishment (DLR), Bunsenstr. 10, D-37073 Göttingen, Germany. 
(Ochadlick, Cho \& Evansmorgis 1992; Peltzer et al. 1992; Vasecky \& Stewart 1982; Gu \& Phillips 1994; Shemdin 1990) as well as from spacecraft (Munk, Scully-Power \& Zachariasen 1987). The observed narrow signatures are independent of the ship's Kelvin wake which has a much wider opening angle. Rather, the signatures are typically confined to the immediate vicinity of the ship's line of passage, a region in which the wake turbulence is believed to interact with surface waves and surface films (also known as surface-active agents or surfactants). It is the damping effect of the surface films on the gravity-capillary waves which is most likely to be responsible for their attenuation near the ship's centreline wake which in turn renders the wake region visible in radar and sun-glint imagery. In-situ surface tension measurements have shown the existence of a persistent sharp discontinuity in the local surface tension near the edges of the far wake (Peltzer et al. 1992). This discontinuity is brought about by the redistribution of surface film by the passing ship. Once displaced, surfactants will typically tend to redistribute themselves on their own which does not seem to occur in the ship's wake. Even wind and wave action are not sufficient to diffuse the surfactant discontinuity. The persistence of this discontinuity suggests the presence of an external force, such as a uniform subsurface flow, to confine surfactants in narrow bands parallel to the ship's wake.

Although no data on the nature of subsurface flow are available to the authors, it is suspected that its origin is a combination of the ship's highly turbulent and bubbly displacement and separated flows as well as its propulsion. As the ship passes it displaces a large volume of water which may result in the formation of a pair of large-scale counter-rotating rollers in the far wake. The evolution of such a flow can be modelled in the laboratory using vortex pairs which are forced to interact with the bounding free surface. This vortex-pair flow has been the subject of many pioneering experimental studies by Sarpkaya \& Henderson (1985), Sarpkaya \& Suthon (1991), Sarpkaya (1992) and Hirsa \& Willmarth (1994) who used either lifting surfaces towed below the surface or counter-rotating flap mechanisms. These studies focused on the impact of vortex pairs on the free surface with regard to surface deformation, vortex depth, and turbulence intensity mainly in the high Froude number range - which is not typical of the far wake. However, the past work, aside from the numerical studies by Dommermuth (1993), did not involve the vorticity field in the description of the interaction processes.

In this work vortex pairs of low Froude number are also generated using a counter-rotating flap mechanism. As a means to capture some of the threedimensional features present in the full-scale ship wake flow, a known spatial perturbation was introduced along the vortex axis at the same time as the pair's formation. This mode of spatial perturbation was chosen over the natural shortand long-wave instabilities since their time scale and growth rate were insufficient to allow controlled and repeatable surface interaction studies. Among the questions studied herein is the mechanism by which the vortex pair may decay in the presence of the free surface and the influence surfactants may have on its decay. In the previous studies these surfactant effects could be considered insignificant in comparison to the higher Froude number dynamics. However, in the far wake region, the effects of surfactants and the associated surface tension gradients begin to dominate the flow as the local Froude number decreases. The overall aim of this study is not to quantify the degree to which specific concentrations of surfactants affect the surface interactions of the vortex pairs. Rather, two cases with widely differing surfactant concentrations are presented in order to 
illustrate two dramatically differing interactions of the vortex pair with the free surface.

\subsection{Vortex pair characterization}

In the characterization of vortex pairs it is helpful to define the associated dimensionless quantities, namely the Reynolds number, $R e$, and the Froude number, Fr. The former is simply the ratio of the vortex pair's circulation, $\Gamma$, and the kinematic viscosity $v: R e=\Gamma / v$. Typically the Froude number is the ratio of a characteristic velocity and the shallow-water wave speed, $c_{o}=(g h)^{1 / 2}$. For Froude numbers $\mathrm{Fr}>1$, the vortex pair's surface flow will become supercritical and may even result in wave formation and breaking. In the cases described here the Froude number is well below 1. A characteristic velocity for the Froude number is the vortex pair centreline velocity, $U_{c l}$, which, by using the point vortex model, is related to circulation through $\Gamma=U_{c l} \pi d / 2$, whereas the propagation velocity is $U_{p}=\Gamma /(2 \pi d)$. The characteristic depth $h$ is taken to be $d / 2$, the distance of the core from the surface ( $d$ is also the distance between the core and its image above the surface). With this choice of characteristic velocity and depth the Froude number can be written in three ways:

$$
F r=\frac{U_{c l}}{\left(g\left(\frac{1}{2} d\right)\right)^{1 / 2}}=\frac{4 U_{p}}{\left(g\left(\frac{1}{2} d\right)\right)^{1 / 2}}=\frac{\Gamma}{\pi\left(g\left(\frac{1}{2} d\right)^{3}\right)^{1 / 2}} .
$$

Although the presence of the factor $\pi$, a constant arising from the point vortex model, would not necessarily seem appropriate in dimensional analysis, experimental data obtained for the vortex pair produce closely matching results for all three definitions of the Froude number if this factor is included. The physical justification for these definitions of $\mathrm{Fr}$ is the experimental observation that the onset of wave breaking and splashing corresponds to $\mathrm{Fr}=1$.

\subsection{The surface boundary condition}

The physically realistic free surface is not only free to vertical excursion (i.e. waves) but almost always has a contamination layer on it whose effects cannot be ignored. These surface-active agents (or surfactants) create a boundary condition on the fluid below that is neither free-slip nor non-slip but rather a combination of both. The reason for the mixed-slip condition is the nonlinear response of the surfactants to flow-induced compression and expansion as there is a strong tendency to restore the film deformations. The restoring nature of the surfactant layer creates a shear force on the adjoining fluid elements which give rise to additional mechanisms of vorticity generation at the free surface.

In the context of describing capillary and gravity waves, Longuet-Higgins (1960, 1992) has shown that, given a steady flow below a shear-free curved surface, vorticity is generated at the surface. Lugt (1987) provided a similar result for vortex/free surface interaction. Based on a local polar-coordinate analysis of the free surface, both theories only treat the steady case whose net vorticity production below the surface remains zero if diffusive effects are excluded. The analysis may be extended to include unsteady effects through the use of an orthogonal curvilinear coordinate reference frame as depicted in figure 1 (see also Rood 1994). The analysis requires two coordinate systems: a global Cartesian coordinate system whose $(x, z)$-plane is aligned with the equilibrium free surface, and a local curvilinear coordinate system that moves with the deforming free surface. For simplicity, it is assumed that the flow locally is two-dimensional such that the radius of curvature associated with the out-of-plane axis $(z)$ is infinite, which makes the $z$-axis and the $\zeta$-axis of the respective coordinate 

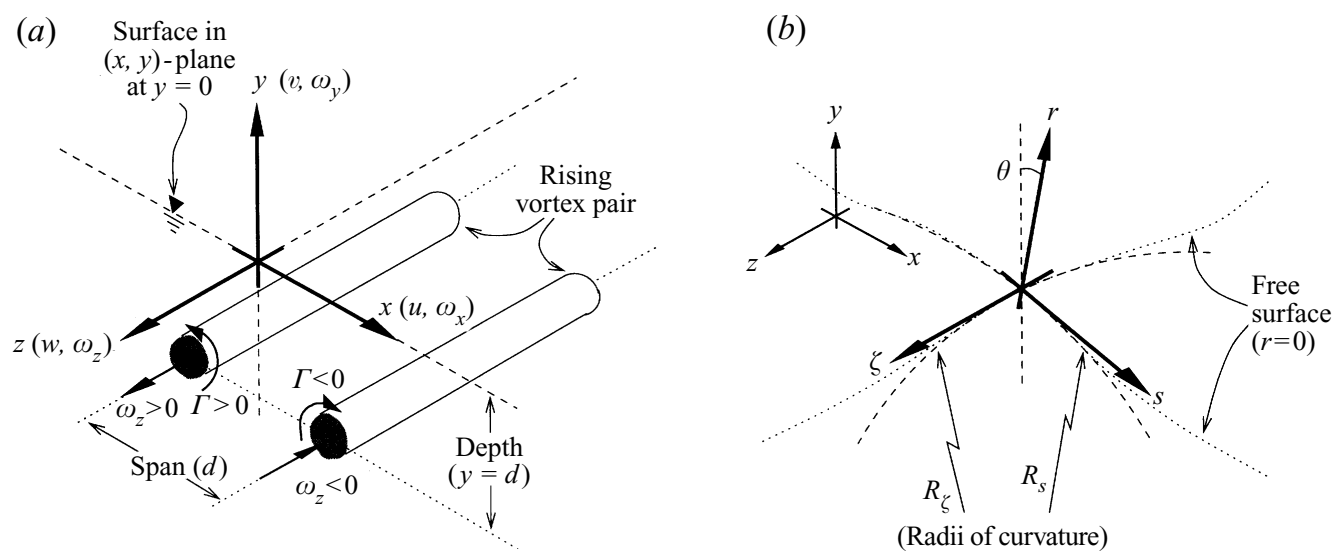

FIGURE 1. The coordinate systems used in the analysis of the vortex/free surface interaction study: (a) global Cartesian coordinate system, (b) local curvilinear coordinate system.

systems parallel. As a result of this, the surface-parallel vorticity component, $\omega_{\zeta}$, is identical to $\omega_{z}$.

The analysis involves a force balance at the interface: that is, the shear created by the adjoining air and gradients in surface tension, $\sigma$, have to be balanced by the shear on the fluid element directly below the interface as the distance $r \rightarrow 0$. Through the substitution of the surface-parallel vorticity component, with $r \rightarrow 0$, into the force balance, an expression for the surface-parallel vorticity at the interface is determined:

$$
\omega_{z}=-\frac{\tau_{A i r}}{\mu}-\frac{1}{\mu} \frac{\partial \sigma}{\partial s}-2 \frac{u_{s}}{R_{s}}+2 \frac{\partial u_{r}}{\partial s} .
$$

The terms on the right-hand side of (1.1) can be described as: $(a)$ a generation of vorticity by wind, $(b)$ vorticity created by gradients in the surface tension on the surface (i.e. the elasticity of the surface layer imposes a shear force on the adjoining fluid), (c) locally created vorticity as the flow follows a curved surface, and $(d)$ vorticity created by unsteady vertical motion of the surface.

Associated with this surface-parallel vorticity is a viscous transport equation at the interface whose derivation can be found in Rood (1994):

$$
-v\left(\frac{\partial \omega_{z}}{\partial r}\right)_{r=0}=\frac{\partial u_{s}}{\partial t}+u_{s} \frac{\partial u_{s}}{\partial s}+\frac{1}{\rho} \frac{\partial P}{\partial s}+g \cos \theta
$$

or

$$
-v\left(\frac{\partial \omega_{z}}{\partial r}\right)_{r=0}=\frac{\mathrm{D} u_{s}}{\mathrm{D} t}+\frac{1}{\rho} \frac{\partial P}{\partial s}+g \cos \theta .
$$

This expression for the viscous flux of surface-parallel vorticity, $\omega_{z}$, suggests that surface-parallel vorticity can be introduced into or removed from the surface by means of a surface acceleration (or deceleration), by a surface inclination or by a pressure gradient along the surface. The viscous flux of vorticity through the surface is instrumental in the formulation of the model describing the experimentally observed vortex connection processes shown later in this paper. 


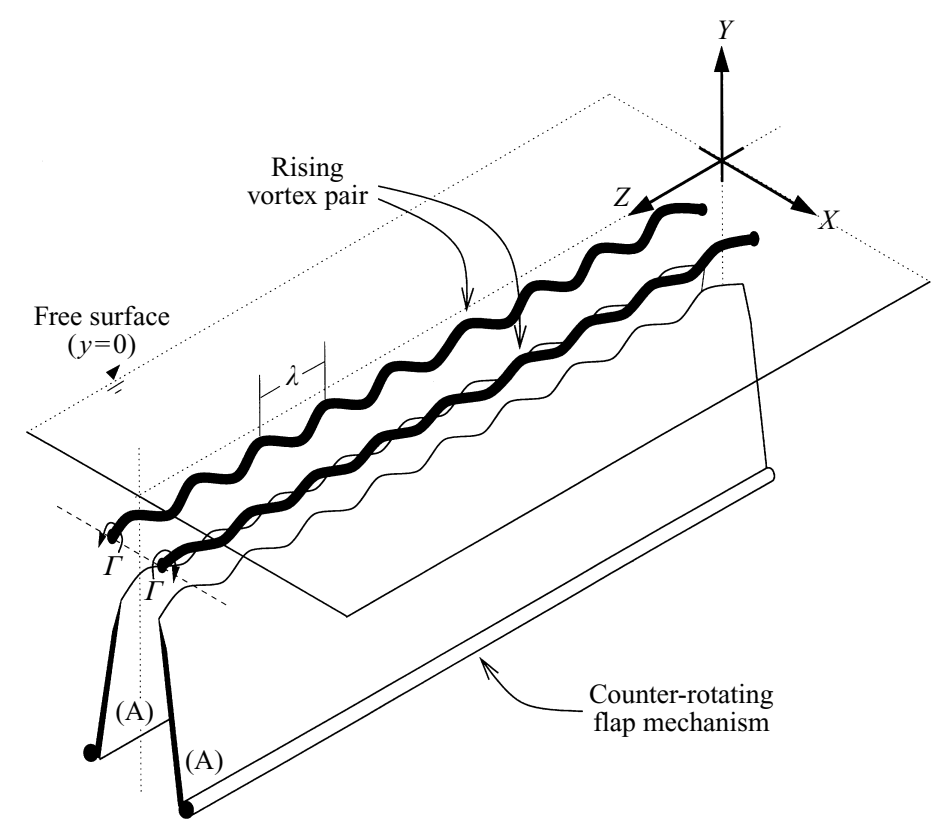

FIGURE 2. The counter-rotation of the flaps (A) produces a pair of counter-rotating vortices that rise toward the surface. The sinusoidal shape of the flap's edge induces a sinusoidal modulation along the length of the rising vortex tubes.

\section{Experimental setup and procedure}

The spatially modulated vortex pairs are generated below the free surface by two counter-rotating flaps as diagrammed in figure 2. The edges of the sharpened flaps approximate a sinusoid with a wavelength, $\lambda=8 \mathrm{~cm}$, and a peak-to-trough distance of $2 a=1.6 \mathrm{~cm}(a / \lambda=10 \%)$. Other earlier experiments used slightly different configurations. These $20 \mathrm{~cm}$ wide and $90 \mathrm{~cm}$ long flaps are made from clear acrylic and are attached to two stainless steel axles of $2.0 \mathrm{~cm}$ diameter. Slippage-free counterrotation is achieved by a steel cable that is wound in a figure-eight fashion between two pulleys on one end of the axles. A linkage attached to one axle is connected to a linear displacement stepper motor.

The shape of the motion profiles executed by the stepper motor is crucial to the nature of the vortex pair created. Dye visualization experiments, as well as initial velocity field measurements with the apparatus, had shown that flap motion profiles consisting of a mostly constant velocity flanked by steep accelerations and decelerations (i.e. a top-hat motion profile) resulted in the formation of a shear layer containing a series of small vortices which rolled up into one large vortex (Willert 1992). This vortex shedding behaviour virtually disappeared for stepper motor motion profiles consisting of only a constant acceleration followed by a constant deceleration of equal magnitude and duration (i.e. a triangular motion profile). The deceleration of the flaps caused the newly formed vortex pair to separate from the flaps while the stopping vortex pair generated during this time was trapped by the closing flaps. These triangular motion profiles were used exclusively for the vortex connection experiments described herein.

The presence of surface-active agents (or surfactants) has dramatic effects on the nature of vortex/free surface interactions (Bernal et al. 1989; Bernal \& Kwon 1989; 
Hirsa \& Willmarth 1994; Tryggvason et al. 1992), so a variety of methods were employed in an effort to reduce their presence. This included the removal of oils and other potential surfactants (such as fingerprints) from the inside walls of the tank and all of the submerged equipment using methanol and/or acetone. The tank then was filled with de-ionized water. To further reduce the buildup of surfactants, the tank was covered and, during the course of the later experiments, was located in a clean-room laboratory. Nevertheless, significant amounts of surfactants of airborne origin as well as from residual contaminants on the equipment collected with time such that a continuously running stand-pipe was needed to draw in the surface layer. Additional surface cleansing/drainage was achieved by using a strong vacuum cleaner to remove the surface layer a few minutes before each experiment. Because the measurement of surface tension, and hence the degree of contamination, could not be measured in-situ but rather by sampling the surface water using a small dish. An empirical test described by Scott (1975) indicated a low degree of contamination: small bubbles that came to the surface from below persisted only for a very short time $(<1 \mathrm{~s})$ before bursting. The presence of surfactants, even in small concentrations, strongly increases the persistence time.

In terms of instrumentation both qualitative as well as quantitative tools were employed. The shadowgraph technique, a flow visualization tool, was the primary means of investigation for many of the flow studies and was used to single out specific cases of interest for further investigation by more quantitative tools such as timeresolved particle image velocimetry (PIV). Shadowgraphs are maps of the surface curvature and are created by the refraction of light rays of an initially parallel beam of light. Refraction at the curved surface causes the light rays to diverge or converge such that bright areas indicate a convex surface profile (surface bumps, wave crests, etc.) while the dark regions are surface depressions. The source of light was a highpowered spatially small light source (Oriel 1000 Watt Xenon arc lamp) which was focused on a pinhole (diameter $\approx 100 \mu \mathrm{m}$ ) such that the emanating light essentially formed a point source of light. This point was placed at the focal point of a spherical mirror $(\mathrm{fl}=60 \mathrm{~cm}, D=20 \mathrm{~cm})$ which resulted in a reflected beam of parallel light rays. The beam was then directed through the tank's glass bottom toward the surface above the vortex generator, ultimately projecting onto a translucent screen above the tank. The illuminated screen itself was imaged using a shuttered video camera $(1 / 125 \mathrm{~s}$ exposure) whose signal was recorded by a laser disk player.

To get further insight into the flow topology and its evolution, quantitative timeresolved measurements were made using the Digital Particle Image Velocimetry (or DPIV) technique (Willert \& Gharib 1991; Willert 1992). This method captures the instantaneous cross-sections of a three-dimensional flow field at fixed time intervals (here $15 \mathrm{~Hz}$ ) and thus produces velocity vector maps of the flow in both space and time. The measurements are performed by illuminating particles suspended in the fluid with a pulsed laser light sheet and recording the scattered light using an electronic camera. By measuring the particles' movement from one frame to the next using statistical methods, the two in-plane velocity components can be estimated by dividing by the known time difference between the illumination pulses and multiplying by the image-to-object magnification factor. The vorticity component normal to the imaged plane can then be approximated from the velocity field using a finite difference scheme. A variety of other quantities such as circulation are obtained by further post-processing the velocity data.

The light sheet illumination was created by spreading the beam from a $5 \mathrm{~W}$ Argon ion laser using a plano-concave cylindrical lens (figure 3). The continuous laser light was shuttered synchronously with the framing rate of the CCD camera 


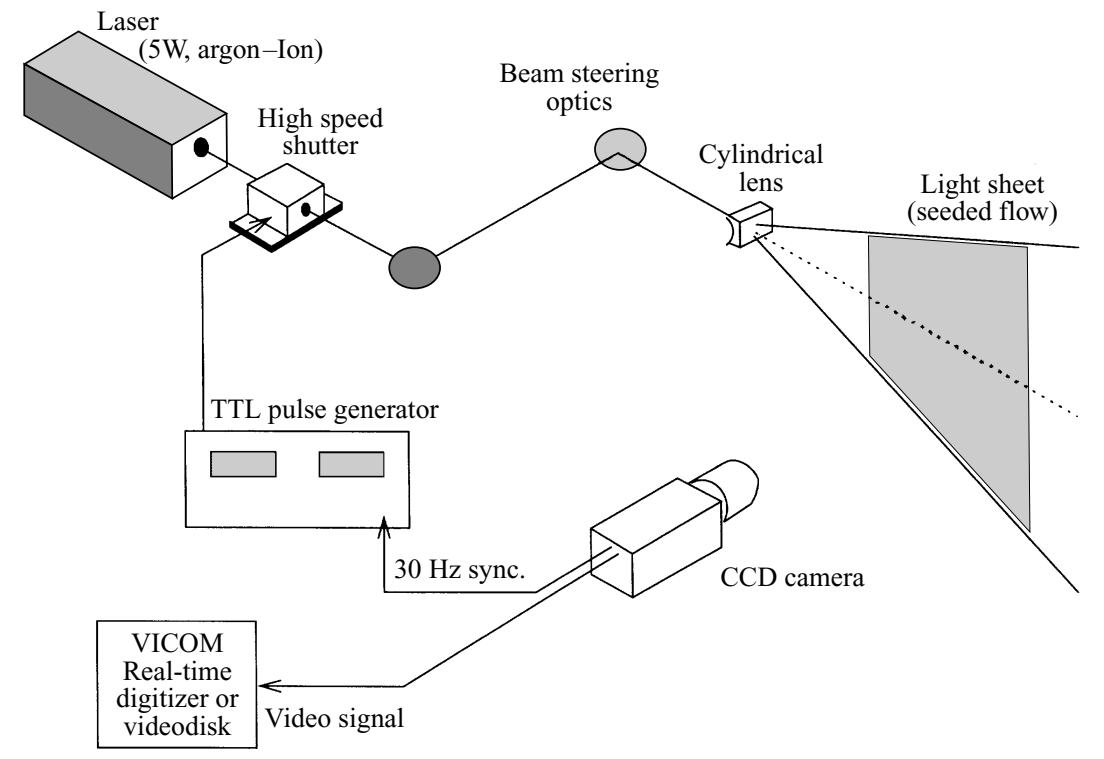

FIGURE 3. Diagram of the DPIV image acquisition system.

(Texas Instruments MC-1134P) with a shutter-open time of only 2 ms per video frame which prevents a streaking of the particle images on the sensor whose exposure time lasts $33.3 \mathrm{~ms}$. The frame store capability of this specific camera allowed the laser light pulse pairs to be supplied asynchronously in $15 \mathrm{~Hz}$ intervals, producing DPIV image pairs with a time delay reduced to $10 \mathrm{~ms}$ instead of the usual $33.3 \mathrm{~ms}$ (i.e. $30 \mathrm{~Hz}$, the camera-framing rate). As a result, the particle displacement between the frame of a DPIV image pair was reduced allowing the measurement of higher velocities at the same magnification factor and also decreasing the likelihood of particles leaving the light sheet through three-dimensional motion.

Because of their availability two different types of seeding particles were used in the course of the experiments. Initially fluorescent neutrally buoyant polymer spheres with a mean diameter of $80 \mu \mathrm{m}$ (special order from the Kodak Chemical Products Division) were used. Less-expensive silver-coated hollow glass spheres with a mean diameter of $10 \mu \mathrm{m}$ (Potters Industries) provided a significantly higher seeding density, whereas the settling effects associated with a slightly higher specific weight were offset by the small size of the particles. The direct reflection off the smaller particles resulted in a scattering cross-section similar to that for the larger fluorescent particles. The specific weight difference also was insufficient to cause a visible migration of the particles away from the vortex core.

The DPIV image pairs were either acquired digitally using a real-time digital video disk as part of an image processing system (VICOM), or in analog form using a Sony laser video disk. Selected frames of the latter were digitized after the completion of the experiments. Aside from a few minor modifications the DPIV image analysis software employed was identical to that presented in Willert \& Gharib (1991).

\section{The vortex pair experiments}

The parameter space associated with the study of vortex pairs which interact with the free surface is very large and involves the vortex circulation, $\Gamma$, the mean 
core separation, $\bar{d}$, the core diameter, the amplitude, $a$, and wavelength, $\lambda$, of the spatial modulation, the formation depth, $h$, the formation time/length scales, the surface boundary conditions, as well as several others. The thrust of the experiments presented here was to record data for two limiting extremes of the surface boundary conditions while keeping the initial conditions of the vortex pairs themselves the same. The first case describes the interaction with a surface from which surfactants have been removed to the highest degree possible by using the methods described in \$2. Surface tension measurements were made on samples taken from the tank using a ring-type surface tensiometer and verified an overall cleanliness of the surface for the duration of the experiment. Nevertheless, surface tension deviations of only fractions of a dyne $\mathrm{cm}^{-1}$ from the 'clean'-water value are sufficient to significantly affect the flow. Incidentally this small difference is on the order of the accuracy and uncertainty in the surface tension measurement technique making it an unreliable indicator for the presence of surfactants. Also, even in the case of a thoroughly cleansed surface, Reynolds ridges, clear indicators of a contaminated surface, appear (see figure 5). Their effect on the primary structure is insignificant though, which is not the case for the more contaminated surface.

In the second case studied herein, the stand-pipe and filtration system were shut off to allow an accumulation of surfactants. Several drops of the surfactant ethylalcohol were also added to the surface. In this case, no effort was made to quantify the amount of surfactants on the surface as the interest here was only to illustrate the effect on the flow. However, samples taken from the tank indicated that the surfactants lowered the surface tension on the order of 10 dyne $\mathrm{cm}^{-1}$.

\subsection{Interactions with 'clean' surfaces}

The surface interactions of rather weak vortex pairs $(1000<R e<8000)$ were visualized with shadowgraphs using several different spatial modulation wavelengths, $\lambda$, and amplitudes, $a$. It was observed that the surface interactions are a function of the flap dimensions $(\lambda, a)$, the flap starting angle, the flap acceleration rates, and the distance, $h$, between the flap edge and the free surface. The flap motion parameters (initial angle and acceleration rate) determine the vortex core size, the mean core separation, $\bar{d}$, the circulation, $\Gamma$, as well as the orbital velocity, $\Omega$, of the orbital plane of the sinusoidally shaped vortex cores in space.

The core size, which was found to depend largely on the flap motion during vortex formation and not on the final vortex circulation, has a direct effect on the type of surface features observed. The shadowgraph sequence shown in figure 4 was created by a vortex pair $(R e=\Gamma / v \approx 4700, F r \approx 0.75)$ that was generated at a constant flap speed flanked by high acceleration/deceleration. The surface features are dominated by striations which were oriented normal to the main vortex axis and connected across the vortex pair. In the numerous trailing vortex studies by Sarpkaya (1992) and Sarpkaya \& Suthon (1991) these features also have been observed and were linked to cross-axis vorticity using flow visualization methods. The vortex pair experiments by Hirsa \& Willmarth (1994) resulted in the appearance of these striations as well. Numerical simulations performed by Dommermuth (1993) topologically link these cross-axis vortices (striations) to the vorticity shed by the spatially modulated primary vortex tubes during their approach to the surface. In all cases, as well as in the present experiments, the final stages of the vortex/free surface interaction are characterized by a connection of the striations to the free surface which is evidenced by small circular black 
(a)

$t=0.40 \mathrm{~s}$

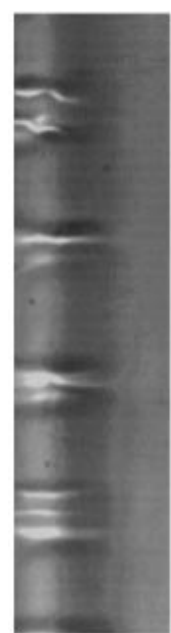

(b)

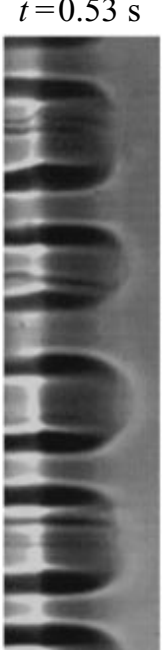

(c) $t=0.60 \mathrm{~s}$ (d)

$$
t=0.70 \mathrm{~s}
$$

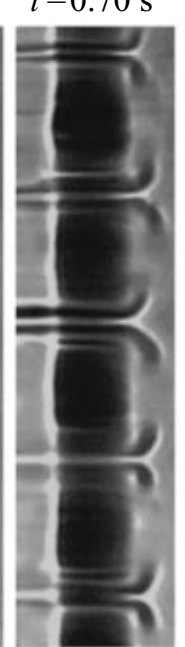

(e)

$t=0.83 \mathrm{~s}$

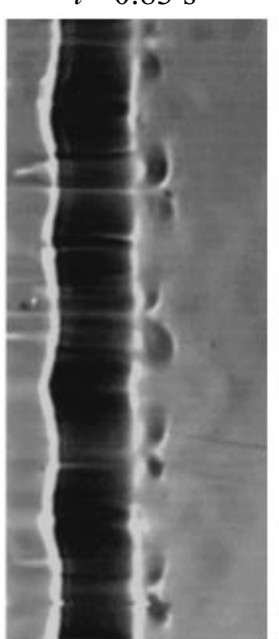

(f)

$t=1.26 \mathrm{~s}$

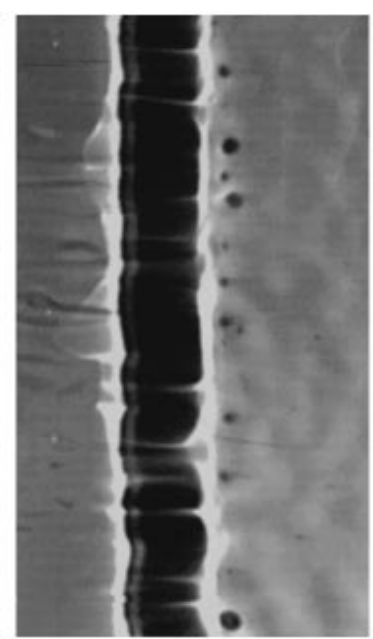

FiguRE 4. Shadowgraph images of one core of a thick core vortex pair $(\operatorname{Re}=\Gamma / v \approx 4700, F r \approx 0.75$, $\lambda=5.5 \mathrm{~cm}, a / \lambda=7.2 \%$ ). Propagation is from the left to the right. The field of view is $25 \mathrm{~cm}$ high.

patches, located upstream of the primary vortex in the shadowgraph images. The link between the surface depression and surface-normal vorticity is further supported by simultaneous shadowgraph and PIV measurements performed by Weigand (1996).

In the data presented so far the underlying main vortex tube is not observed to connect to the surface. Since the present study emphasizes the interaction of the primary vortical structure with the free surface, the vortex formation parameters were varied until a repeatable connection of the primary vortex to the surface as in the shadowgraphs of figure 5 could be observed. The subsequent velocity field (DPIV) measurements indicated that the pattern shown in figure 5 only arises if the mean core separation, $\bar{d}$, is less than twice the spatial modulation amplitude, or $\bar{d}<2 a$. An explanation for this behaviour may be that the core modulation amplitude has to be comparable to the mean propagation distance of the vortex core below the surface which forces portions of the core to be in very close proximity to the surface. This proximity is a prerequisite for a direct interaction between the vortex and the surface. This interaction is further enhanced by a smaller core size, which, due to its highly concentrated vorticity, reduces the time scale of the interaction.

The cross-axis vortices (striations) which were observed in the previous experiments (e.g. figure 4) only appear faintly in figure $5(a)$. Instead the shadowgraph images are dominated by the shape of the spatial modulation on the primary vortex tube. Here the orbital plane of the sinusoidal modulation appears to be approximately parallel to the surface. Figure $5(b)$ shows three sites - doublets of dark circular patches which will be referred to as a 'vortex connection' in the following Sections. The middle site is in the early stages of the connection, whereas the upper site is nearly fully developed. With increasing time these surface depressions increase in area but decrease in surface curvature since their darkness is reduced. This indicates that the pressure gradient toward the connected vortex core is decaying which may indicate a larger core size at the surface. 
(a) $t=2.00 \mathrm{~s}$

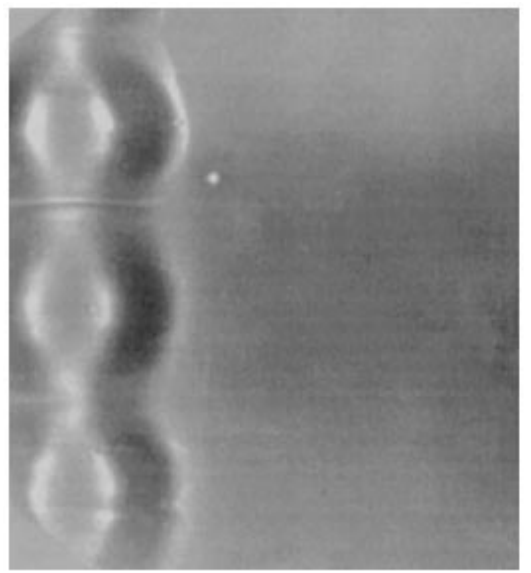

(c) $t=3.83 \mathrm{~s}$

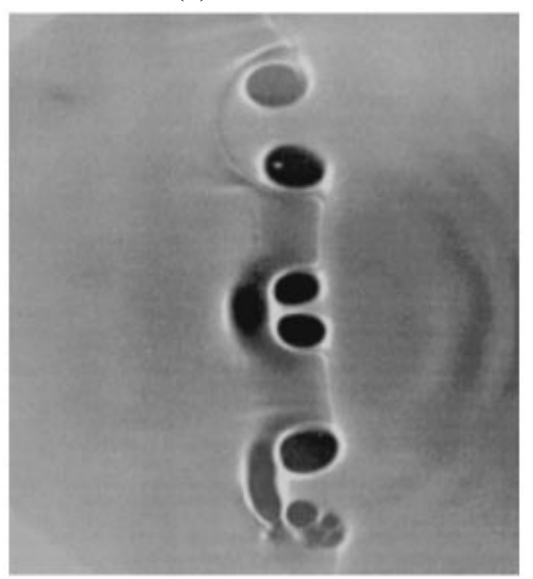

(b) $t=3.20 \mathrm{~s}$

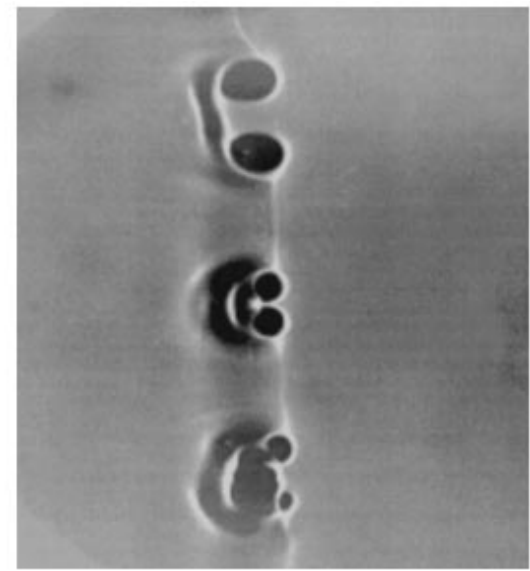

(d) $t=4.56 \mathrm{~s}$

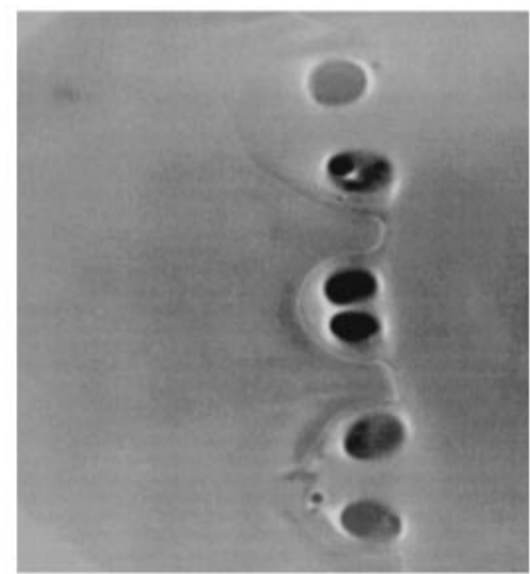

FIGURE 5. A spatially modulated vortex pair $(R e=\Gamma / v \approx 5000, F r \approx 0.3, \lambda=8 \mathrm{~cm}, a / \lambda=10 \%)$ interacting with a relatively 'clean' surface. The core of the vortex moves from the left to the right within the image. The field of view is $25 \mathrm{~cm}$ high.

Another feature of the connection process is the formation of weak surface waves as seen in figure 5(c). Also, the structures that connected first tend to dominate the ones appearing later as evidenced by the small size of the central connection site in figure $5(d)$. An explanation for this may be that the connection process changes the global vortex dynamics in the surrounding field.

Quantitative evidence for the connection process was obtained for the flow described above using the DPIV technique described in $\$ 2$. As shown in figure 6 , three planes were necessary to fully capture the repeatable flow field shown in the previous shadowgraphs: plane A intersects the cores in the middle of the connection site $(Z=0)$, coincident with one of the peaks along the flap's sinusoidal edge; plane B is parallel to but removed from plane A by $Z=\lambda / 2=4 \mathrm{~cm}$, thus intersecting the region between the connection sites; and plane $\mathrm{C}$ consists of the subsurface flow plane at approximately $y=-2 \mathrm{~mm}$ below the surface. Each of these planes was imaged separately but the good repeatability between the runs allowed a direct alignment in both space and time. 


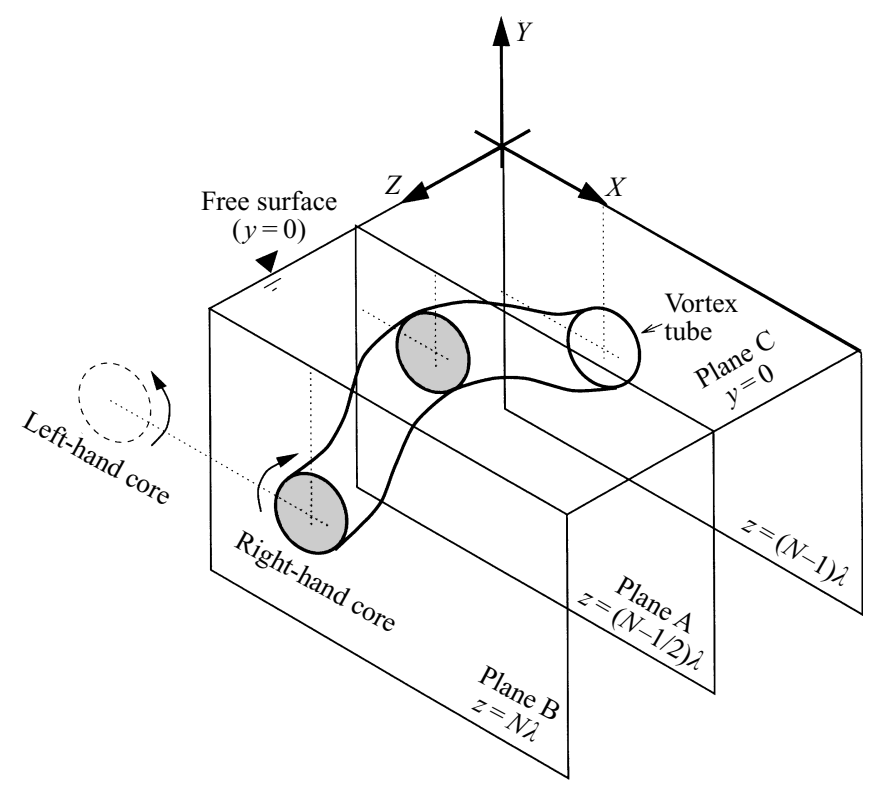

FIGURE 6. Cross-sections that were captured with the DPIV measurement technique.

Velocity and vorticity measurements of the core cross-sections are shown in figures 7 and 8 and are complemented by the subsurface measurements of figure 9. In plane A (figures 7 and 8, left-hand columns) the vortex core approaches the surface $(y=0)$, flattens alongside it and loses significant amounts of vorticity. Within $2 \mathrm{~s}$ the original vortex structure is depleted to a relatively small and weak core. A very different behaviour is seen for the parallel plane B (figures 7, and 8, right-hand columns), removed half a wavelength from plane A. Here the core never reaches the surface and remains intact even while vorticity is being lost in the neighboring plane A.

The subsurface flow data (figure 9) shows the connection process even more clearly; the shape of the velocity field at $t=2.06 \mathrm{~s}$ has a close resemblance to the shadowgraph image of figure 5(a). The spatial modulation of the underlying vortex core is clearly evident. During the connection process from $t \approx 2.0 \mathrm{~s}$ to $t \approx 3.0 \mathrm{~s}$, the surface velocity at $Z=0$ decreases locally as the core comes very close to the surface (figure 7, left-hand column), which suggests that a local loss of vorticity parallel to the surface is directly related to a local surface deceleration.

The total amount of gained or lost vorticity is reflected in the integral measurement of circulation around the vortex cores which is plotted with respect to time in figure 10 . The measurements were made using the paths of constant vorticity (typically $\omega= \pm 1 \mathrm{~s}^{-1}$ ) to integrate the velocity field in approximation to the path integral $\Gamma=\int_{S} \boldsymbol{u} \cdot \mathrm{d} \boldsymbol{s} \equiv \iint_{A} \omega \mathrm{d} \boldsymbol{A}$. Here it is important to point out that the circulation in plane A decreases in direct proportion to the increase of circulation of the vortical structures in the subsurface plane $\mathrm{C}$. The circulation of the core region in plane $\mathrm{B}$ remains unaffected throughout the circulation (vorticity) transfer.

Given the experimental data, a sketch of the final vortex tube arrangement can be proposed and is shown in figure 11. In this drawing, a small vortex tube remains and trails behind the advancing U-shaped vortices and accounts for the residual vortex observed in plane A. The existence of this residual vortex tube can also be seen in the shadowgraph images of figure 5 . 

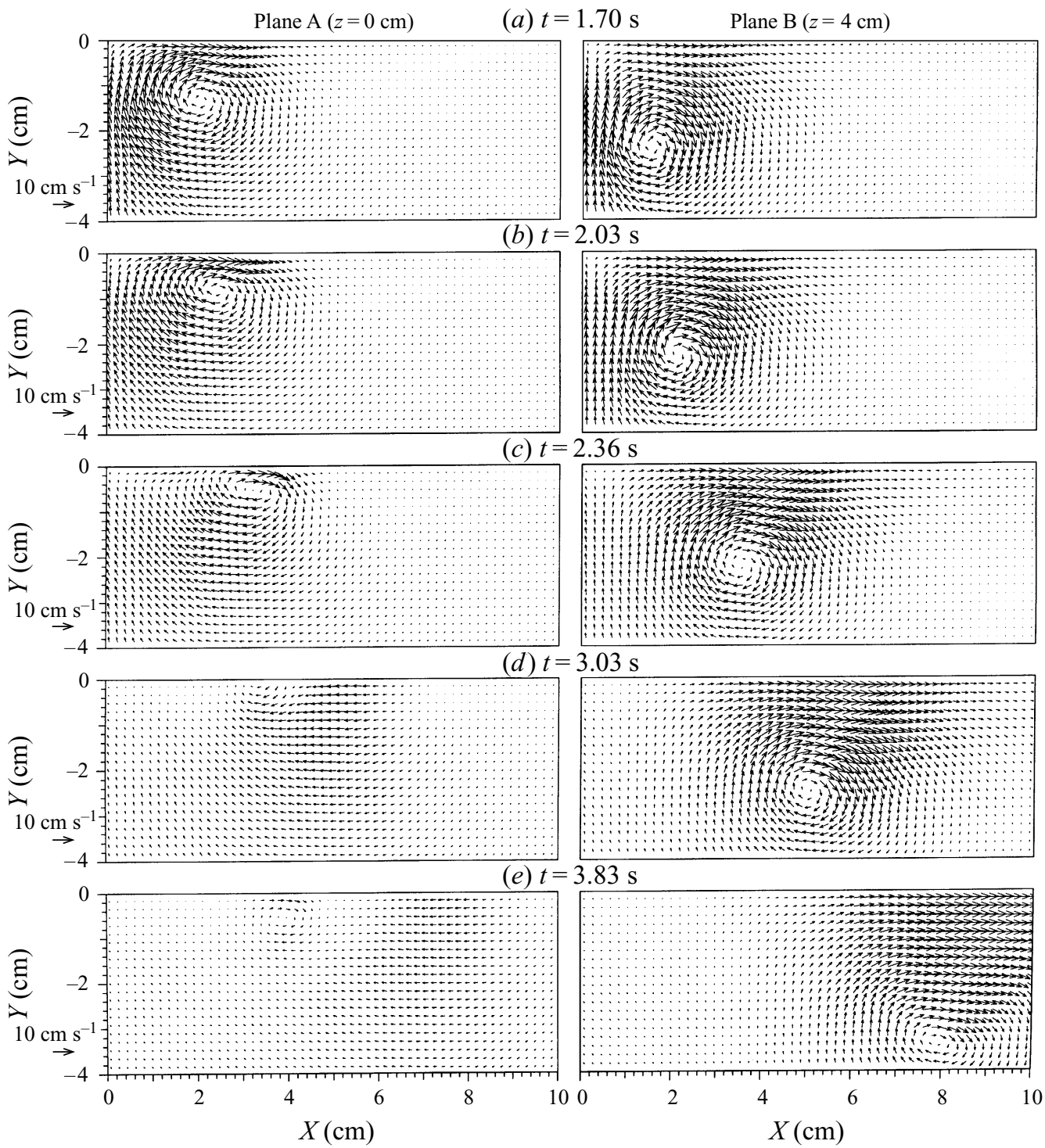

FIgURE 7. Velocity fields in the cross-sectional planes $Z=0$ (left) and $Z=4 \mathrm{~cm}$ (right) at $R e=\Gamma / v \approx 6000, \mathrm{Fr} \approx 0.3$.

\subsection{The influence of surface contamination}

The connection process described in the previous Section is very sensitive to the presence of surfactants. The connections ceased to appear even for a seemingly small reduction in surface tension compared to clean water on the order of $0.5 \mathrm{dyne} \mathrm{cm}^{-1}$. This value is on the order of the measurement uncertainty of the ring-type surface tension measuring device. Figure 12 shows the interaction under the same initial conditions as in figure 5 shown in the previous Section. In this case however the surface cleaning procedures (stand-pipe, vacuuming of the surface) were omitted which resulted in the rapid formation of a surfactant layer. Additional drops of the surfactant ethyl alcohol significantly lowered the surface tension $\left(O\left(5-10\right.\right.$ dyne $\left.\left.\mathrm{cm}^{-1}\right)\right)$. 

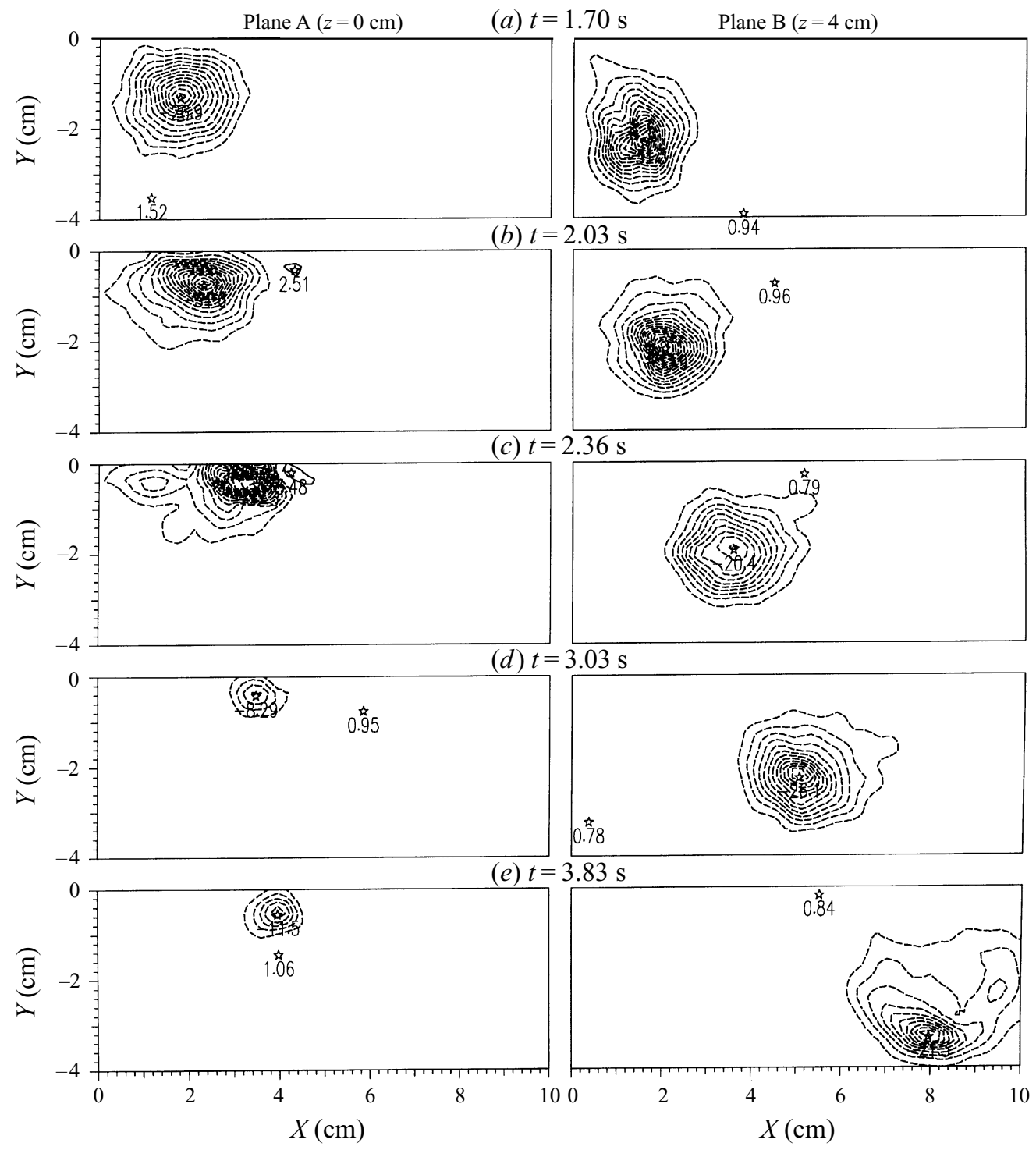

FIGURE 8 . Vorticity fields in the cross-sectional planes $Z=0$ (left) and $Z=4 \mathrm{~cm}$ (right) at $R e=\Gamma / v \approx 6000, F r \approx 0.3$ (contour levels: ..., $\left.-4 \mathrm{~s}^{-1},-2 \mathrm{~s}^{-1}, 2 \mathrm{~s}^{-1}, 4 \mathrm{~s}^{-1}, \ldots\right)$.

While shadowgraph images of the previous 'clean' surface case clearly showed the sinusoidal core shape below the surface at $t=2.00 \mathrm{~s}$, no such signature is observable in figure 12. Rather, a bright line forms on the outer edge of the rising vortex pair. This line is known as the Reynolds ridge (Scott 1982) and essentially forms at a discontinuity of surface contamination. In this case, the up-welling (fresh) water at the centre (to the left of the line) is much cleaner than in the region to the right which was initially stagnant. The Reynolds ridge moves along with the advancing vortex and later deforms to the sinusoidal shape of the underlying vortex tube. Strong connection events as described in the previous Section do not appear in this case. Instead small circular depressions form to the right of the Reynolds ridge in a periodic fashion similar to those in figure 5. These surface depressions also suggest the presence of 

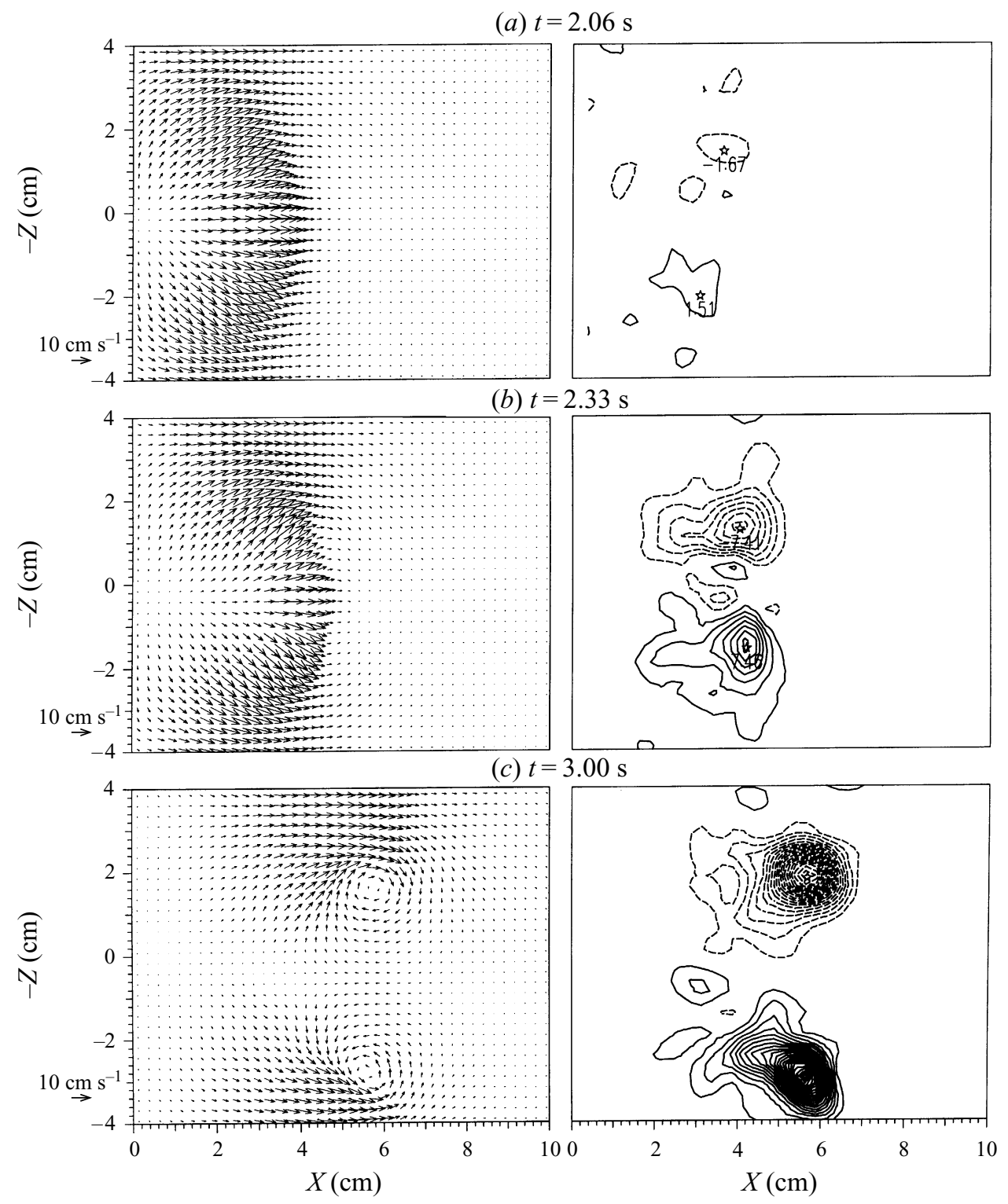

FIGURE 9. Velocity (left) and vorticity fields (right) in the plane $1 \mathrm{~mm}$ below the surface (plane C) at $R e=\Gamma / v \approx 6000, F r \approx 0.3$ (contour levels: ..., $-2 \mathrm{~s}^{-1},-1 \mathrm{~s}^{-1}, 1 \mathrm{~s}^{-1}, 2 \mathrm{~s}^{-1}, \ldots$ ).

surface-normal vorticity and may be a result of a connection process similar to that described before. The subsequent time-resolved DPIV measurements, conducted in analogy the previous 'clean' surface case (Figure 6), captured the associated flow field.

Velocity and vorticity measurements of the core cross-sections are shown in figures 13 and 14 . The cross-sectional vorticity fields of the vortex cores of planes A and B (figure 14) show the appearance of secondary vorticity below the surface at a very early time while the vortex pair is still approaching. The location of maximum vorticity in these structures, above and slightly outside of the main vortex, seems to coincide with the location of the Reynolds ridge in the shadowgraph images. The velocity data 


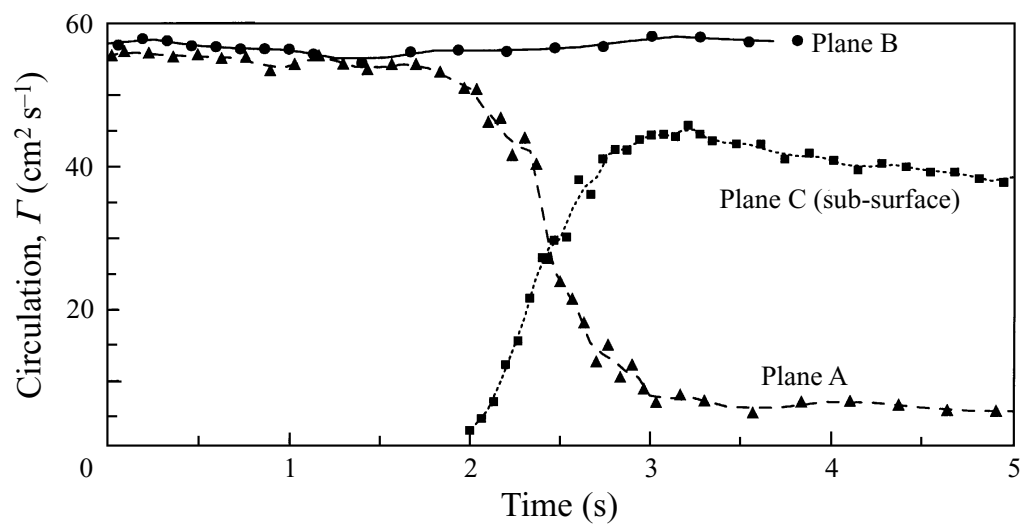

Figure 10. Circulation as a function of time for the vortex pair interacting with a 'clean' surface $(R e=\Gamma / v \approx 6000, F r \approx 0.3)$.

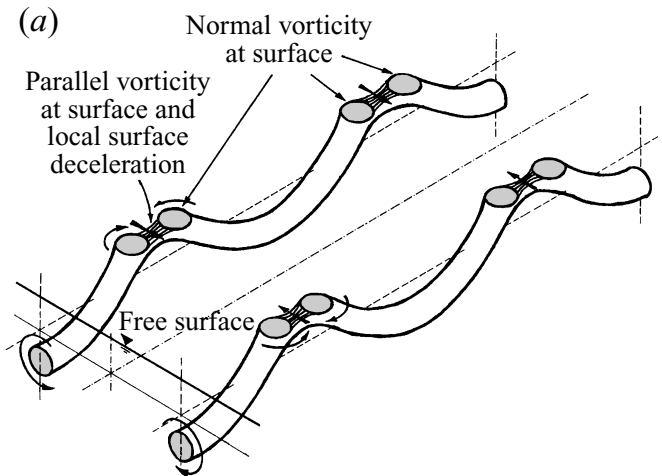

(b)

FIGURE 11. Vortex tube arrangement during and after the interaction with a clean surface. The final configuration $(b)$ shows a series of $U$-vortices connected by residual vortex tubes.

(figure 13) show that the surface velocity changes abruptly in this area redirecting the underlying flow downward - the Reynolds ridge behaves as a stagnation line leading to the formation of a separated flow region below the surface. The velocity measurements of the subsurface plane $\mathrm{C}$ in figure 15 actually show a flow reversal in front of the main vortex tube. In the later stages only a very weak velocity field remains at the surface although the primary vortex tube below the surface remains largely intact. The secondary vorticity accumulates into a rather large structure with significant circulation (figure 16) and is able to divert the main vortex in a downward motion (figures $13 d$, and $14 d$, left), also referred to in the literature as vortex rebound (Bernal et al. 1989; Tryggvason et al. 1992; Hirsa \& Willmarth 1994).

Neither of the two cores in figure 14 shows the dramatic loss of vorticity (and circulation) as in the 'clean' surface case of figure 8. The circulation data of figure 16 show a gradual decay in time which is attributable to the cross-diffusion of oppositesigned vorticity. The circulation of the secondary vortex is detectable more than $1 \mathrm{~s}$ prior to the initiation of the connection (circulation transfer) process observed for the 'clean' surface case. The circulation of the vortical structures in the subsurface plane (plane $\mathrm{C}$ ) has a similar growth rate to the secondary vortices below the surface (planes A and B) which leads to the conclusion that they are part of the same vortical structure. A diagram of the proposed vortex tube arrangement is shown figure 17 . 
(a) $t=2.00 \mathrm{~s}$

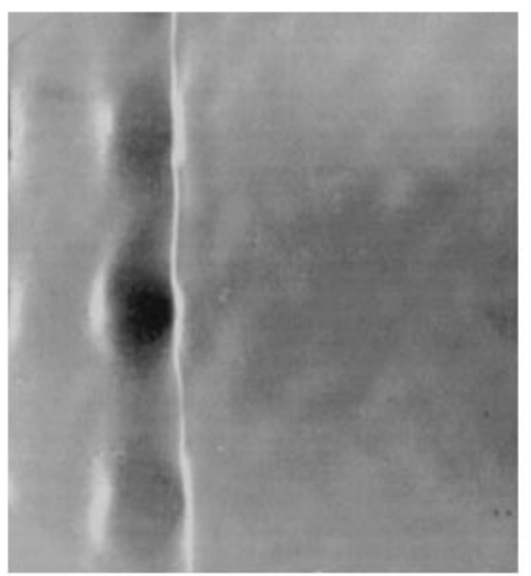

(c) $t=3.50 \mathrm{~s}$

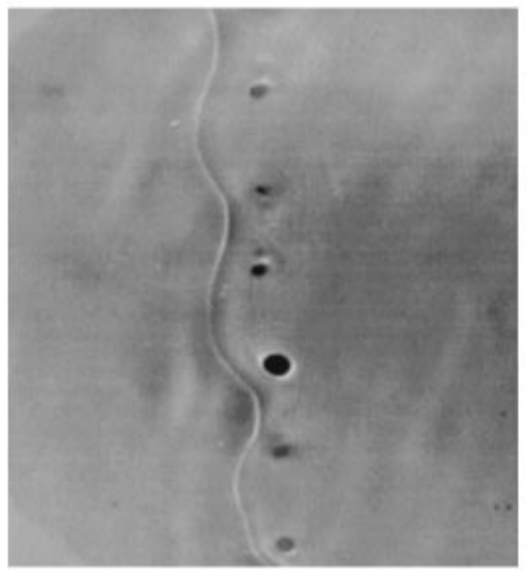

(b) $t=2.73 \mathrm{~s}$

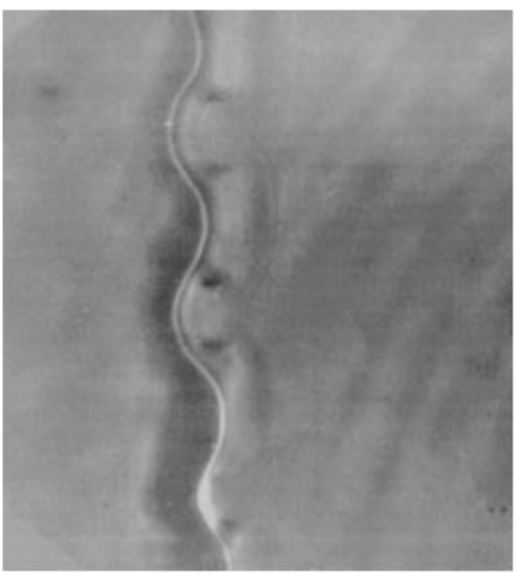

(d) $t=4.50 \mathrm{~s}$

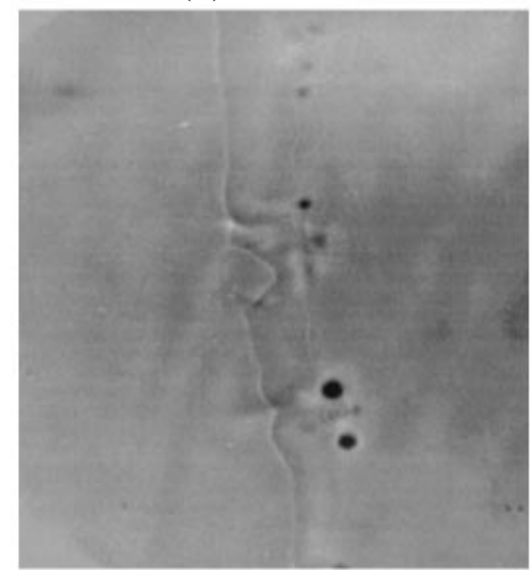

FIGURE 12 . A spatially modulated vortex pair $(\operatorname{Re}=\Gamma / v \approx 5000, F r \approx 0.3, \lambda=8 \mathrm{~cm}, \lambda / a=10 \%)$ interacting with a contaminated surface. The core of the vortex moves from the left to the right with the image. The field of view is $25 \mathrm{~cm}$ high.

\section{Discussion}

In the previous Section, the interactions of vortex pairs with both a relatively clean as well as a highly contaminated free surface were highlighted. Under a certain set of conditions the vortex pair, much like a vortex ring approaching the surface at an angle (Bernal \& Kwon 1989; Weigand 1996), will locally 'connect' by removing the vorticity from the vortex tube closest to the surface to form two parallel lines of U-vortices as shown in figure 11. The DPIV measurements indicate that a necessary condition for the connection is that the high-vorticity vortex core is brought very close to the interface such that it may locally 'lose' its vorticity to the surface. For both the vortex rings and the spatially modulated vortex pairs it is the global flow topology which brings the vortex core close to the surface and thereby forces an interaction with the surface. As the circulation measurements in figure 10 clearly show, the primary vortex core disappears almost entirely in the plane of the connection (plane A, figure 8, left-hand column) - the vortex pair is broken into smaller segments which tend to decay more rapidly than a uniform straight core. 

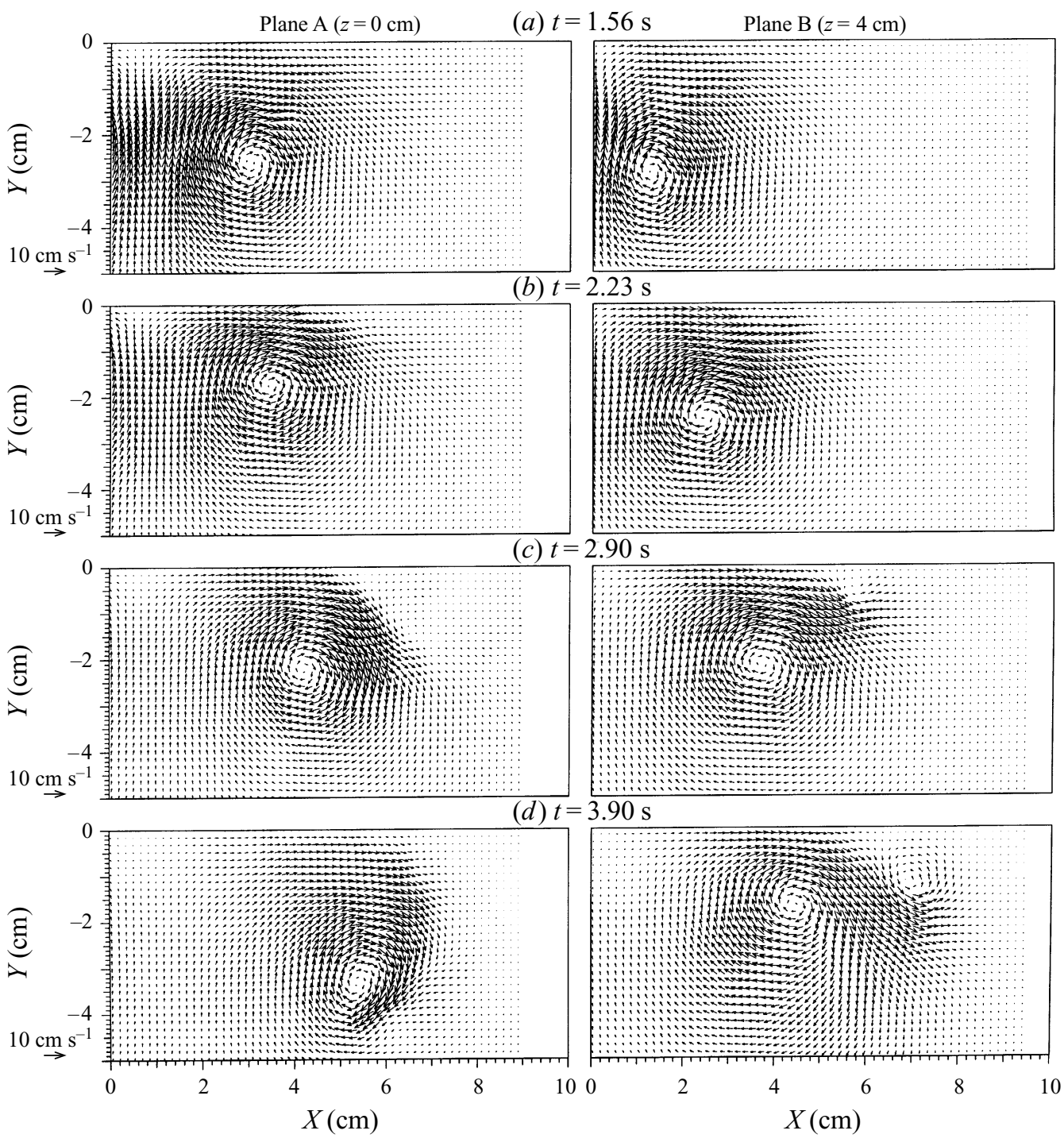

FIGURE 13. Velocity fields in the cross-sectional planes $Z=0$ (left) and $Z=4 \mathrm{~cm}$ (right) for the vortex interaction in the presence of increased surface contamination $(R e=\Gamma / v \approx 6000, F r \approx 0.3)$.

The mechanism of this connection can be described with the expression for the viscous flux of vorticity through the surface given earlier:

$$
-v\left(\frac{\partial \omega_{z}}{\partial r}\right)_{r=0}=\frac{\partial u_{s}}{\partial t}+u_{s} \frac{\partial u_{s}}{\partial s}+\frac{1}{\rho} \frac{\partial P}{\partial s}+g \cos \theta
$$

Although the shadowgraph technique relies on surface deflection to produce an image containing dark and bright patterns, these deflections were too small to be observed in the side-view DPIV recordings. Therefore the term $g \cos \theta$ can be considered to be secondary in the vortex/surface interaction process and thus can be neglected. Since the surface also exhibits only very small differences of elevation the pressure gradient term $\partial P / \partial s$ is also of minor significance here, but may be of importance in other free-surface flows such as the hydraulic jump. The only 

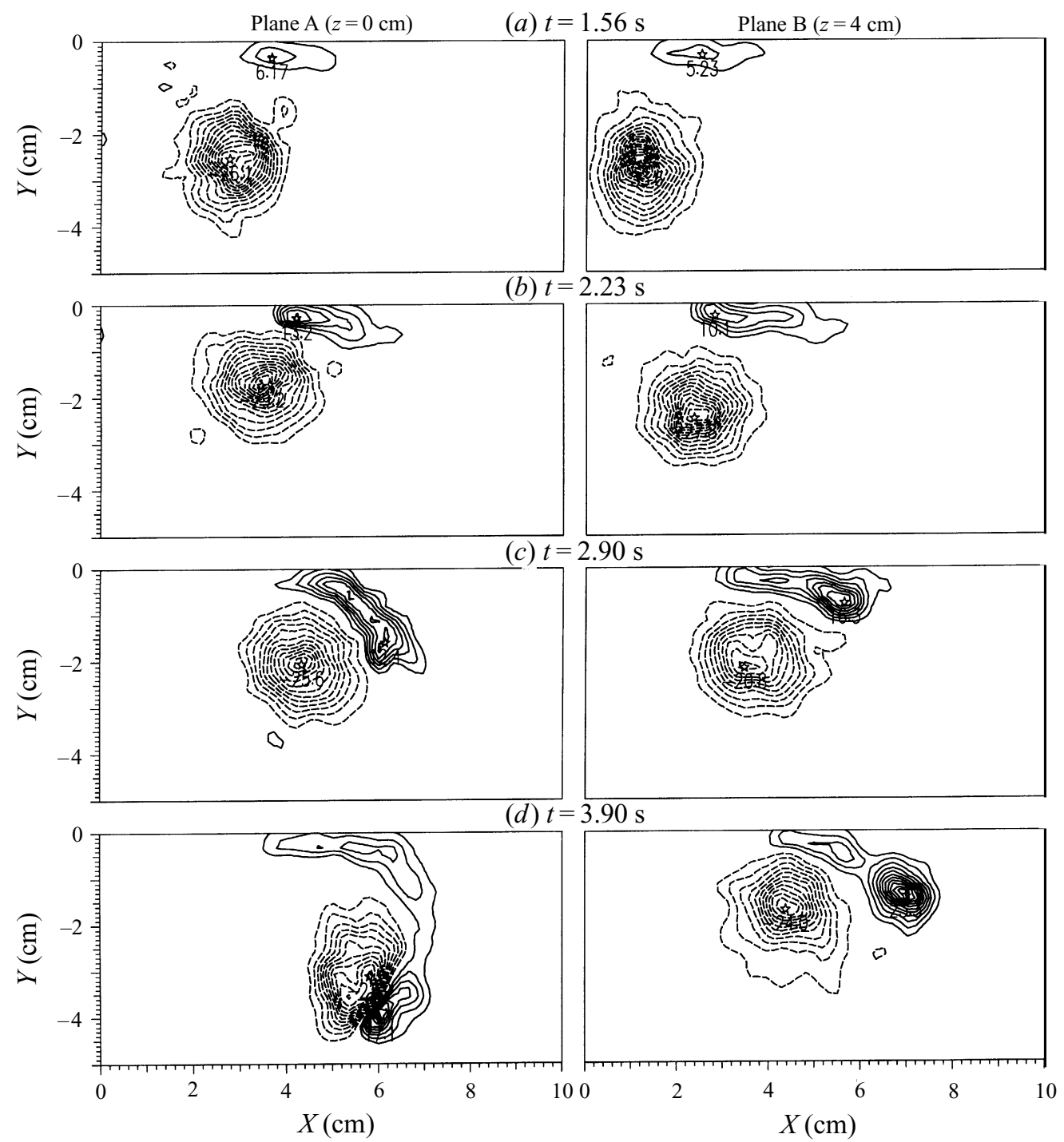

FIGURE 14. Vorticity fields in the cross sectional planes $Z=0$ (left) and $Z=4 \mathrm{~cm}$ (right) for the vortex interaction in the presence of increased surface contamination $(R e=\Gamma / v \approx 6000, F r \approx 0.3$, contour levels: ..., $\left.-4 \mathrm{~s}^{-1},-2 \mathrm{~s}^{-1}, 2 \mathrm{~s}^{-1}, 4 \mathrm{~s}^{-1}, \ldots\right)$.

significant terms on the right-hand side of (4.1) is that of surface acceleration, $D u_{s} / D t=\partial u_{s} / \partial t+u_{s} \partial u_{s} / \partial s$. This suggests that any loss of surface-parallel vorticity through the surface results in a surface acceleration (or deceleration).

The best test for this hypothesis would be to compute the remaining two terms in expression (4.1) and see whether they match during the connection process. This however is hampered by some crucial drawbacks in the DPIV measurement technique employed. First of all, DPIV is a spatially averaging measurement technique: that is, the measured flow field is a lowpass filtered representation of the actual flow field. As a consequence of this, strong velocity gradients will be smoothed proportionally to the size of the interrogation window - roughly $5 \times 5 \mathrm{~mm}$ for the measurements shown herein. The computation of gradients from the spatially averaged vorticity field near 


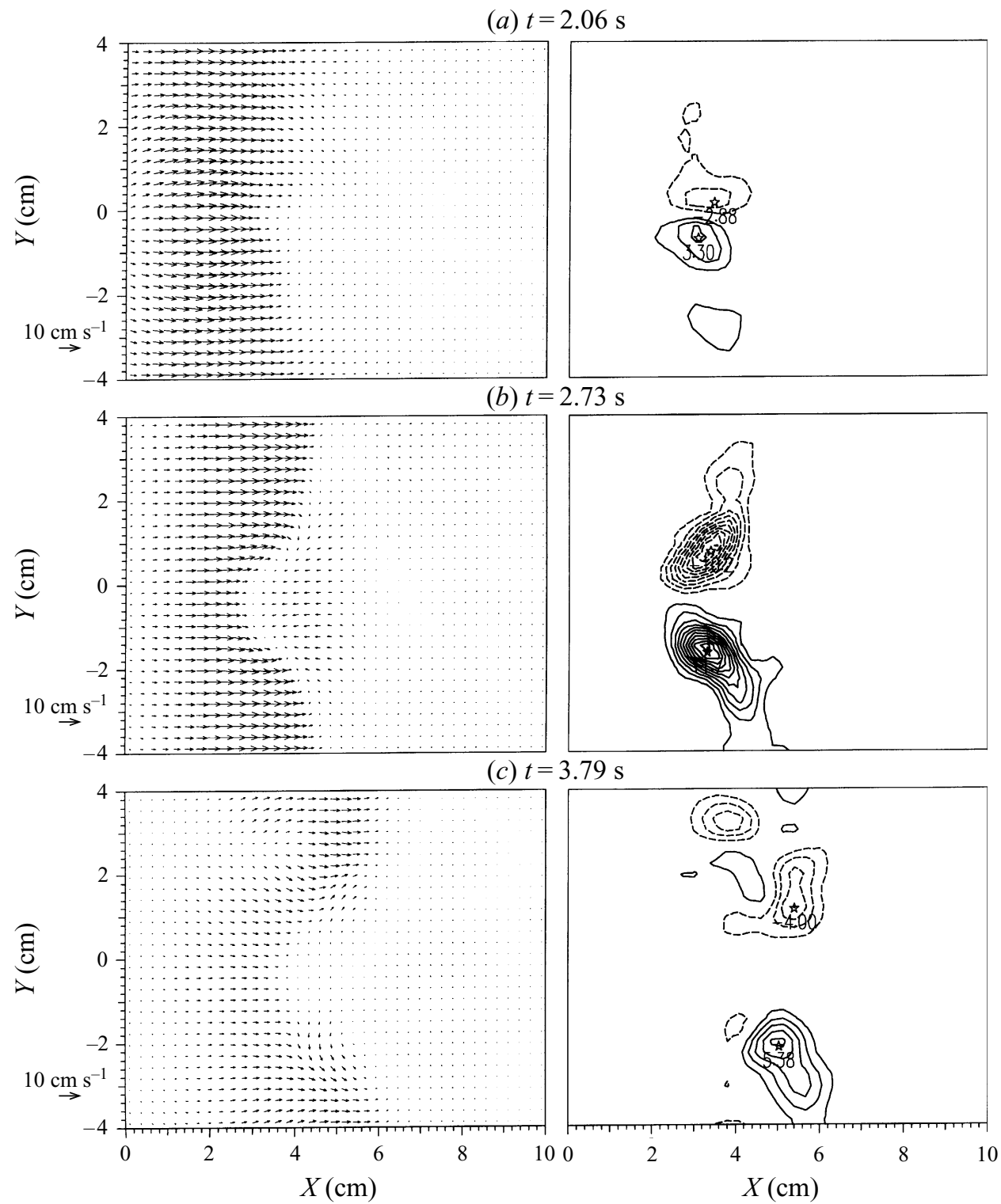

Figure 15. Velocity (left) and vorticity fields (right) in Plane C $1 \mathrm{~mm}$ below the surface $\left(R e=\Gamma / v \approx 6000, F r \approx 0.3\right.$, Contour levels: ..., $\left.-2 \mathrm{~s}^{-1},-1 \mathrm{~s}^{-1}, 1 \mathrm{~s}^{-1}, 2 \mathrm{~s}^{-1}, \ldots\right)$.

and at the surface thus becomes meaningless even if the additional errors due to the second differentiation, i.e.

$$
\frac{\partial \omega}{\partial r}=\frac{\partial}{\partial r}\left(\frac{\partial u_{s}}{\partial r}-\frac{\partial u_{r}}{\partial s}+\mathrm{HOT}\right)
$$

could be accounted for. The first term on the left-hand side of equation (4.1), $\left(\partial \omega_{z} / \partial r\right)_{r=0}$, thus cannot be computed reliably.

The second restriction presented by the DPIV technique is its limited temporal resolution. With the described setup the DPIV time slices were obtained from double 


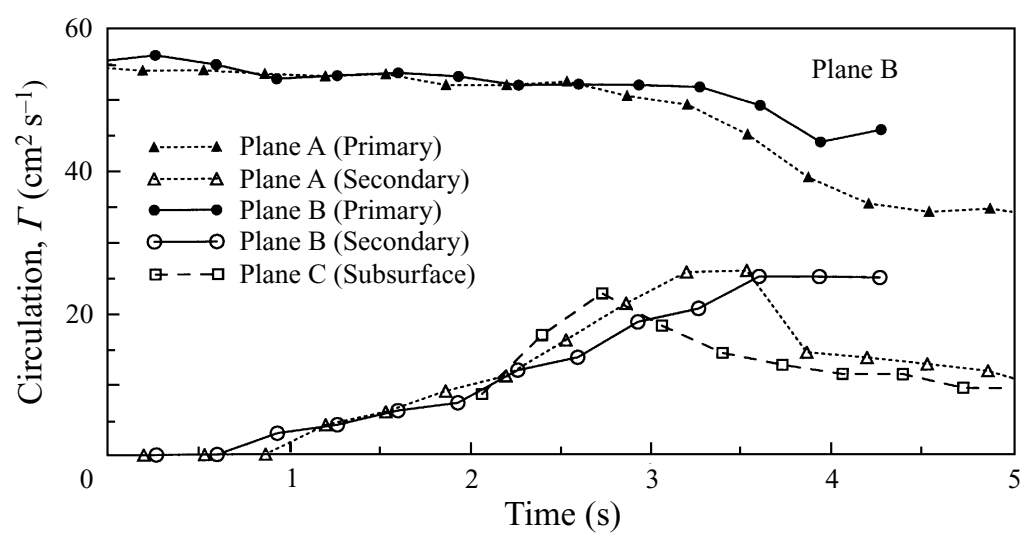

FIGURE 16. Circulation as a function of time for the vortex pair interacting with a surfactant-rich surface $(R e=\Gamma / v \approx 6000, F r \approx 0.3)$.
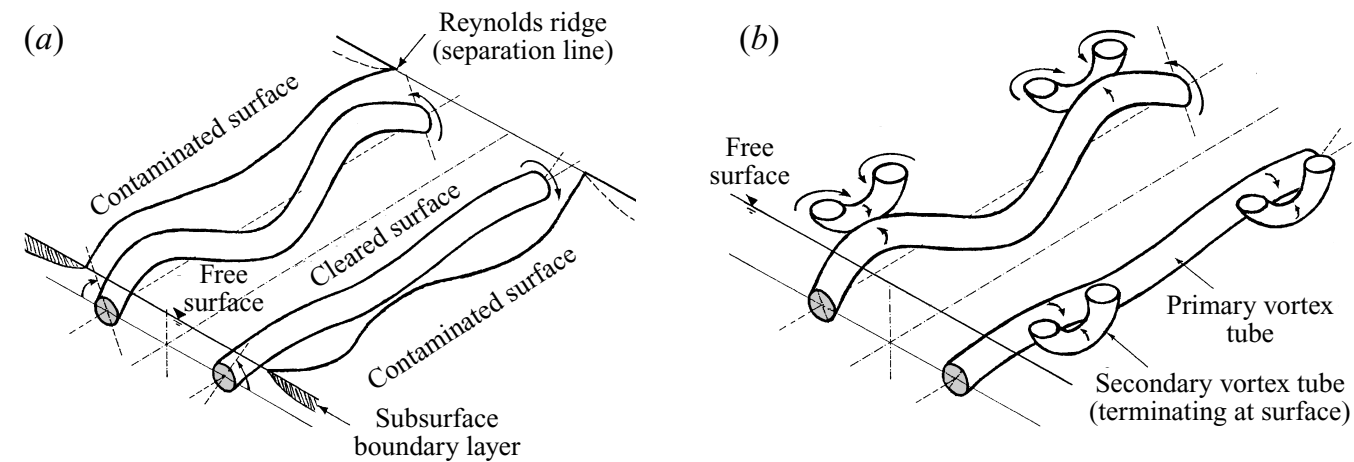

FIGURE 17. Increased amounts of surface contamination result in the formation of a subsurface boundary layer above the approaching vortex pair $(a)$. The opposite-signed vorticity in this boundary layer is concentrated into vortex tubes that terminate at the surface $(b)$ in an arrangement similar to that shown in figure 11.

light pulses with $10 \mathrm{~ms}$ separation in $15 \mathrm{~Hz}$ cycles which is sufficient to provide good representations of instantaneous velocity fields at these time steps. However, the temporal spacing of $66 \mathrm{~ms}$ (e.g. $15 \mathrm{~Hz}$ sampling rate) is too large to allow the computation of the acceleration from one PIV data set to the next. The advective term, $\boldsymbol{u} \cdot \nabla \boldsymbol{u}$, in the substantial derivative,

$$
\frac{\mathrm{D} \boldsymbol{u}}{\mathrm{D} t}=\frac{\partial \boldsymbol{u}}{\partial t}+\boldsymbol{u} \cdot \nabla \boldsymbol{u},
$$

cannot be separated from the actual particle acceleration given in (4.1) and becomes increasingly significant as the time difference increases. Therefore neither the acceleration of the surface nor the flux of surface-parallel vorticity, $\omega_{z}$, can be extracted from the velocity field directly.

Although the DPIV technique is not the method of choice for single-point measurements, it is very suitable for the estimation of integral quantities from the velocity fields such as circulation, entrainment and volume (mass) flux. Rather than computing the local vorticity gradient at the surface, the effect of the surface velocity itself was studied. The following observation can be made: the integration of (4.1) along a line on the surface expresses the net flux of vorticity per unit depth over the 

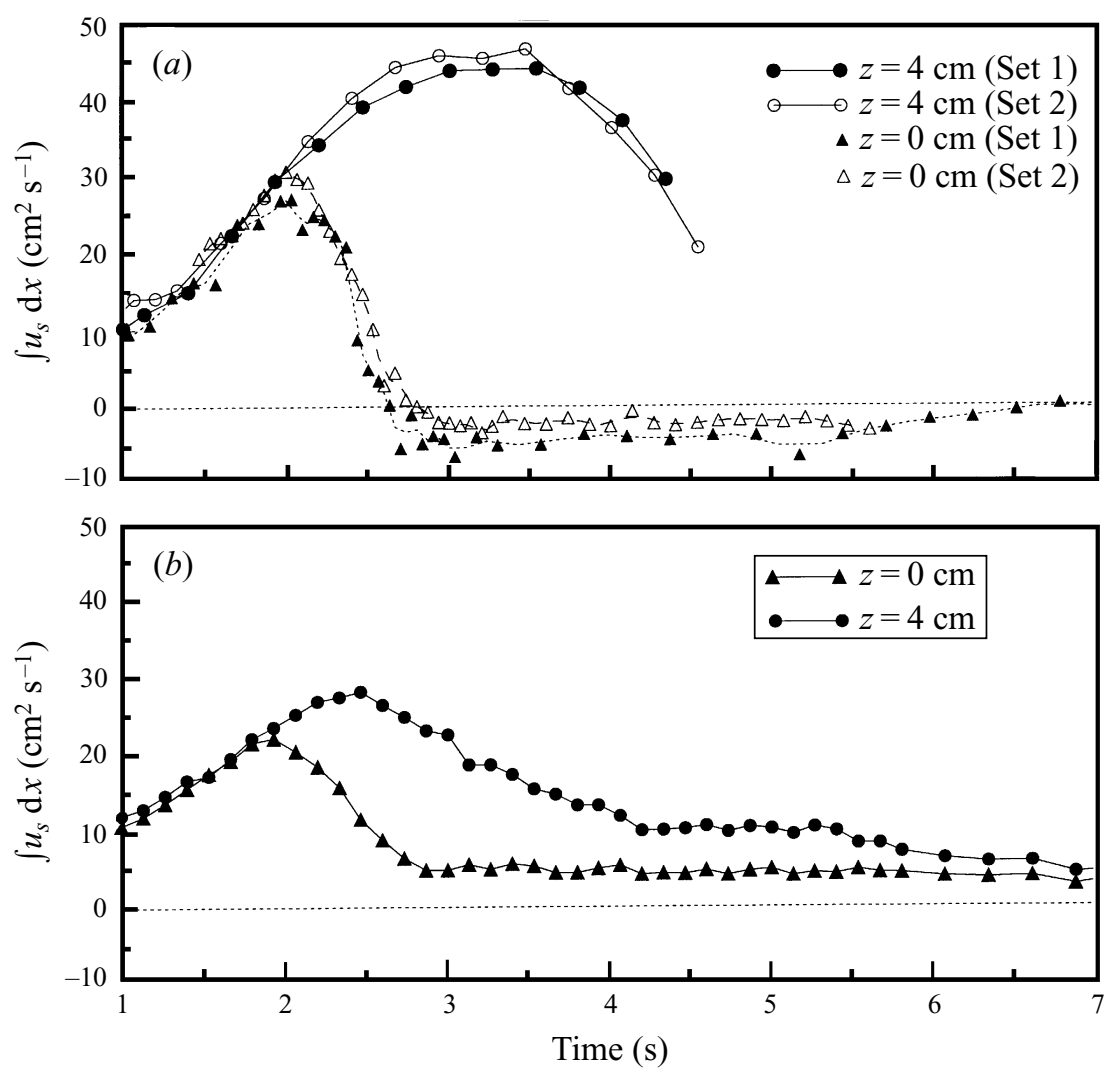

FIGURE 18. Temporal evolution of surface velocity integral $\int u_{s} \mathrm{~d} x$, for a surface with minimal surface contamination $(a)$ and with increased surface contamination $(b)$. The integral is obtained by integration from $x=0$ to the right-hand image boundary $(x=15 \mathrm{~cm})$.

surface. A further integration in time gives a measure of how much circulation, $\Delta \Gamma$, is lost through this line on the surface. For the acceleration term in (4.1), $\partial u_{s} / \partial t$, the calculation is equivalent to integrating the surface velocity, $u_{s}=u_{x}$, from the symmetry plane at $x=0$ over one of the cores to a point located infinitely far away:

$$
\Delta \Gamma=\int_{\tau=t_{0}}^{t} \int_{s=0}^{\infty} \frac{\partial u_{s}}{\partial \tau} \mathrm{d} s \mathrm{~d} \tau=\int_{s=0}^{\infty} \int_{\tau=t_{0}}^{t} \frac{\partial u_{s}}{\partial \tau} \mathrm{d} \tau \mathrm{d} s=\int_{s=0}^{\infty} u_{s} \mathrm{~d} s .
$$

Figure 18(a) shows the result of the surface velocity integration at the line bisecting the connection area $(z=0)$ and the line between the connection areas $(z=\lambda / 2=$ $4 \mathrm{~cm}$ ). Between the connection sites, that is, above the core that remains intact, the integral grows during the approach of the vortex to a maximum at $t \approx 3.0 \mathrm{~s}$, whereafter it decays. This decay is caused by the vortex leaving the field of view for which the integration boundary at $x=12 \mathrm{~cm}$ is no longer sufficient. With an infinite boundary, this value would remain nearly constant.

On the other hand, the surface line integral bisecting the connection region $(z=0)$, above the disappearing core, shows that the same initial growth rate is observable during the approach phase of the vortex. Coincident with the core deformation and circulation loss starting at $t \approx 2.0 \mathrm{~s}$, the surface circulation, $\Delta \Gamma$, drops sharply within $1.2 \mathrm{~s}$ and remains at the level of $\Delta \Gamma \approx-5.0 \mathrm{~cm}^{2} \mathrm{~s}^{-1}$. The difference between the two integration paths is approximately $50 \mathrm{~cm}^{2} \mathrm{~s}^{-1}$ which is equivalent to the vorticity 
loss of the core in plane A (see figure 10). This clearly suggests that all vorticity lost from the vortex core is converted to a local surface acceleration above the core.

A completely different behaviour of the vortex tubes is observed when the surface is contaminated, that is, the surface boundary conditions are modified. The presence of even a small amount of surfactants and the associated small reduction in surface tension $\left(\sigma_{\text {clean }}-\sigma_{\text {dirty }}=O\left(1\right.\right.$ dyne $\left.\left.\mathrm{cm}^{-1}\right)\right)$ prevents a connection of the type described above. Instead, the induced surface velocity field of the approaching vortex pair redistributes the surfactants whose own restoring force leads to a subsurface shear force which, in turn, is accompanied by a subsurface boundary layer. It is the oppositesigned vorticity from this boundary layer that keeps the concentrated vorticity of the core from reaching the surface and thus prevents the initiation of the connection process.

During the interaction with the contaminated surface a significant amount of opposite-signed vorticity is generated and wrapped around the primary core where cross-diffusion of vorticity will eventually consume the vortex pair. Some surfacenormal vorticity does appear but is connected to the secondary vortex below the surface. These structures have a shorter lifespan than the larger U-vortices described in $\$ 3.1$, which may have something to do with the straining motion of the stronger primary core.

Figure 18(b) shows the results of the line integration proposed in (4.2). The initial growth rate during the approach of the vortex pair is similar to the data reported in figure $18(a)$. At time, $t \approx 2 \mathrm{~s}$, the two integrals depart from one another, which may be explained by the fact that the core section at $z=0$ is closer to the surface and more rapidly produces opposite-signed secondary vorticity. The maximum difference between the integrals is on the order of $18 \mathrm{~cm}^{2} \mathrm{~s}^{-1}$. For the minimally contaminated surface (figure 18a), this discrepancy was explained by the fact that the surface is locally decelerated by the diffusion of the core through the surface. In this case vorticity is introduced into the surface by a backward acceleration of the surface in front of the main vortex tube. In effect, the sign of the integral is the same in both cases such that a knowledge of the flow geometry below the surface is required in determining the sign of introduced or removed vorticity through the surface.

The presence of surfactants also has an effect on the lifespan of the vortex pair. In the 'clean' surface case, the formation of U-vortices from the spatially modulated vortex tube further decreases the stability of the resulting flow field since the Uvortices have a tendency to interact among each other. The surface contamination delays the breakup of the main structure because the subsurface boundary layer prevents a local flux of primary vorticity through the surface. The scavenging of secondary vorticity from the subsurface boundary layer by the main core and the subsequent cross-diffusion between the two structures takes place on a longer time scale than in the case of primary vortex tube connection. As a result, the main core stays intact below the surface and only gradually loses its circulation. With regard to an increased Reynolds number flow, one may picture a large-scale vortex tube with a high total circulation whose increased depth below the surface allows little interaction between the main core and the surface while the secondary vorticity of the contaminant-generated subsurface boundary layer can only slowly dissipate the main structure. This high Reynolds number, but low Froude number, flow may exist in the far field of a ship and may partially explain the persistence of the uniform surface features, such as the surfactant bands, near the centreline of the far wake. 


\section{Summary}

In correspondence to the findings of Sarpkaya (1992), Sarpkaya \& Suthon (1991) and Hirsa \& Willmarth (1994), the impingement of longitudinal vortex tubes on the free surface results in the appearance of striations which are associated with cross-axis vorticity that formed from vorticity shed by the primary vortex structure. In the later stages of the flow, these cross-axis vortices are seen to reattach to the surface and generally leave the underlying primary vortex unaffected. If, however, the vortex formation parameters are chosen such that the core diameter is reduced and the lobes of the spatially modulated primary vortex tube can be brought sufficiently close to the surface, the primary vortex tube has been experimentally shown to connect normally to the shear-stress-free uncontaminated free surface. This connection is retarded through the presence of a surfactant layer whose deformation by the subsurface flow leads to a shear stress and the associated creation of opposite-signed secondary vorticity. Located above and upstream of the advancing primary vortex tube, this secondary vorticity prevents the lobes of the main vortex structure from transferring their surface-parallel vorticity to the surface, which is essential in the connection process. Rather, portions of the separated secondary vortex structure are seen to connect normally to the surface. Because the cross-diffusion between secondary and primary vorticity takes place on a longer time scale than the connection of the primary vortex to a clean surface, the primary vortex tube can persist longer below a contaminated surfactant-laden surface.

The physical model for the connection process stipulates that the surface-parallel vorticity is introduced to or removed from the surface in direct correspondence to a surface acceleration (or deceleration). The available velocity data from the various cross-sections of the flow were first used to obtain circulation estimates of the vortex tubes and verified that circulation lost in one cross-section of the flow reappeared in the orthogonal surface-parallel plane in an one-to-one fashion. The differential expression for the viscous flux of vorticity could not be obtained directly because the spatially smoothed PIV velocity data do not permit a reliable evaluation of differentials, especially at the surface itself where high gradients are to be expected. The PIV data are more suited to recovering line or spatial integrals of the flow field. Thus, the amount of surface-parallel vorticity (or circulation) removed through the surface was estimated using line integrals of the surface velocity along the surface $\left(\int_{x} u_{s} \mathrm{~d} x\right)$. The temporal evolution of these integral quantities shows that, for the connection observed in surfactant-free cases, all surface-parallel vorticity was converted to a deceleration of the surface. In the case of the surfactant-laden free surface, the surface is accelerated in a similar fashion all across the span of the primary tube.

This work was sponsored by the Office of Naval Research under a URI Grant (N00014-92-J-1610).

\section{REFERENCES}

Bernal, L. P., Hirsa, A., Kwon, J. T. \& Willmarth, W. W. 1989 On the interaction of vortex rings and pairs with a free surface for varying amounts of surface active agent. Phys. Fluids A 1 2001-2004.

Bernal, L. P. \& Kwon, J. T. 1989 Vortex ring dynamics at a free surface. Phys. Fluids A 1 449-451.

Dommermuth, D. G. 1993 The laminar interactions of a pair of vortex tubes with a free surface. $J$. Fluid Mech. 246, 91-115.

Gu, D. \& Phillips, O. M. 1994 On narrow V-like ship wakes. J. Fluid Mech. 275, 301-321. 
Hirsa, A. \& Willmarth, W. W. 1994 Measurements of vortex pair interaction with a clean or contaminated free surface. J. Fluid Mech. 259, 25-45.

Longuet-Higgins, M. S. 1960 Mass-transport in the boundary-layer at a free oscillating surface. $J$. Fluid Mech. 8, 293-306.

Longuet-Higgins, M. S. 1992 Capillary rollers and bores. J. Fluid Mech. 240, 659-679.

Lugt, H. J. 1987 Local flow properties at a viscous free surface. Phys. Fluids 30, 3647-3652.

Munk, W. H., Scully-Power, P. \& Zachariasen, F. 1987 Ships from Space. Proc. R. Soc. Lond. A 412, 231-254.

Ochadlick, A. R., Cho, P. \& Evansmorgis, J. 1992 Synthetic aperture radar observations of currents colocated with slicks. J. Geophys. Res. 97, 5325-5330.

Peltzer, R. D., Griffin, O. M., Barger, W. R. \& Kaiser, J. A. C. 1992 High-resolution measurement of surface-active film redistribution in ship wakes. J. Geophys. Res. 104, 245-258.

Rood, E. P. 1994 Free-surface vorticity. In Fluid Vortices (ed. S. Green), Ch. 18. Kluwer.

SARPKAYA, T. 1992 Three-dimensional interactions of vortices with a free surface. AIAA Paper 92-0059.

Sarpkaya, T. \& Henderson, D. O. 1985 Free surface scars and striations due to trailing vortices generated by a submerged lifting surface. AIAA Paper 85-0445.

SARPKAYA, T. \& Suthon, P. 1991 Interaction of a vortex couple with a free surface. Exps. Fluids 11, $205-217$.

ScotT, J. C. 1975 The preparation of water forsurface-clean fluid mechanics. J. Fluid Mech. 69, 339-351.

ScotT, J. C. 1982 Flow beneath a stagnant film on water: the Reynolds ridge. J. Fluid Mech. 116, $283-296$

Shemdin, O. H. 1990 Synthetic aperture radar imaging of ship wakes in the Gulf of Alaska. $J$. Geophys. Res. 95, 16319-16338.

Tryggvason, G., Abdollahi-Alibeik, J., Willmarth, W. W. \& Hirsa, A. 1992 Collision of a vortex pair with a contaminated free surface. Phys. Fluids A 4, 1215-1229.

VASECKy, J. F. \& STEWART, R. H. 1982 The observation of ocean surface phenomena using imagery from the Seasat synthetic aperture radar: An assessment. J. Geophys. Res. 87, 3397-3430.

Weigand, A. 1996 Simultaneous mapping of the velocity and deformation field at a free surface. Exps. Fluids 20, 358-364.

WILLERT, C. E. 1992 The interaction of modulated vortex pairs with a free surface. PhD thesis, Dept. of AMES, University of California, San Diego.

Willert, C. E. \& Gharib, M. 1991 Digital particle image velocimetry. Exps. Fluids 10, 181-193. 